\title{
THE PRODUCT FORMULA FOR STIEFEL-WHITNEY HOMOLOGY CLASSES
}

\author{
STEPHEN HALPERIN AND DOMINGO TOLEDO ${ }^{1}$
}

ABSTRACT. We give a combinatorial proof of the formula for the Stiefel-Whitney homology classes of the product of two Euler spaces. Some relevant facts on ordered triangulations are also included.

Let $X$ be a locally finite, $n$-dimensional polyhedron. $X$ is called an integral Euler space (resp. mod 2 Euler space) if for all $x \in X$ the local Euler characteristic $\chi(X, X-x)=(-1)^{n}($ resp. $\equiv 1(\bmod 2))$. If $K$ is a triangulation of $X$ (always assumed compatible with the PL structure of $X$ ), we denote its first barycentric subdivision by $K^{\prime}$. If $a$ is a vertex of $K^{\prime},|a|$ is the dimension of the corresponding simplex of $K$. Note that the vertices of $K^{\prime}$ are naturally ordered by the inclusion of simplices in $K$.

For $p=0,1, \cdots, n$, the $p^{\prime}$ th Stiefel chain of $K^{\prime}$ is the chain (infinite if $X$ is not compact)

$$
s_{p}\left(K^{\prime}\right)=\sum_{a_{0}<\cdots<a_{p}}(-1)^{\left|a_{0}\right|+\cdots+\left|a_{p}\right|}\left\langle a_{0} \cdots a_{p}\right\rangle \in C_{p}\left(K^{\prime}, \mathbf{Z}\right) .
$$

This is just the sum of all $p$-simplices of $K^{\prime}$, with appropriate signs. $s_{0}\left(K^{\prime}\right)$ is an integral cycle whose homology class represents $\chi(X)$ if $X$ is compact and connected.

$X$ is a mod 2 Euler space if and only if all the Stiefel chains are mod 2 cycles [3]. The homology class of $s_{p}\left(K^{\prime}\right)$ is then independent of the triangulation $K$ (cf. [1]) and is called the $p^{\prime}$ th Stiefel-Whitney homology class of $X, w_{p}(X) \in H_{p}\left(X, \mathbf{Z}_{2}\right)$.

$X$ is an integral Euler space if and only if $\partial s_{p}\left(K^{\prime}\right)=\left(1+(-1)^{n-p}\right) s_{p-1}\left(K^{\prime}\right)$ [2]. In this case we get integral classes $w_{p}(X) \in H_{p}(X, \mathbf{Z})$ when $n-p$ is odd, and $w_{p}(X)$ is the Bockstein of $w_{p+1}(X) \in H_{p+1}\left(X, \mathbf{Z}_{2}\right)$.

If $X$ is a smooth manifold, Whitney showed that $w_{p}(X)$ is the Poin-

Received by the editors August 8, 1973.

AMS (MOS) subject classifications (1970). Primary 57 C99.

1 Supported in part by National Science Foundation grant GP-36418 and NRC A 8047. 
caré dual of the usual Stiefel-Whitney cohomology class $w^{n-p}(X)[4]$.

Now if $X$ and $Y$ are integral (or mod 2) Euler spaces, then so is $X \times$ $Y$. The main purpose of this note is to give a combinatorial proof of the product formula for these classes. Here $\times: H_{*}(X) \otimes H_{*}(Y) \rightarrow H_{*}(X \times Y)$ denotes the cross-product in homology:

Theorem. Let $X, Y$ be mod 2 Euler spaces. Then

$$
w_{p}(X \times Y)=\sum_{r=0}^{p} w_{r}(X) \times w_{p-r}(Y) .
$$

We prove this formula in $\$ 1$, assuming some facts on ordered triangulations that are proved in $\$ 2$. With these facts we also get as an easy corollary the product formula for the integral classes:

Corollary. Let $K$ and $L$ be triangulations of integral Euler spaces $X$, $Y$ of dimension $m, n$. Then, if $m+n-p$ is odd, $\Sigma_{r=0}^{p}(-1)^{m r} s_{r}\left(K^{\prime}\right) \times s_{p-r}\left(L^{\prime}\right)$ is an integral cycle that represents $w_{p}(X \times Y) \in H_{p}(X \times Y, Z)$.

J. Milnor has a different combinatorial proof of the product formula. There is also an argument due to C. McCrory and D. Sullivan that reduces it to the well-known formula for manifolds via "resolutions" of Euler spaces.

For other properties of the Stiefel-Whitney homology classes see [1].

1. Proof of the Theorem. Let $K$ and $L$ be triangulations of $X$ and $Y$, and let $K \times L$ denote the cell complex whose cells are the product of a simplex in $K$ with a simplex in $L$. The vertices of its first barycentric subdivision $(K \times L)^{\prime}$ are the pairs $(a, b)$ where $a$ is a vertex of $K^{\prime}$ and $b$ is a vertex of $L^{\prime}$, and are ordered by $(a, b)<\left(a^{\prime}, b^{\prime}\right)$ if $a \leq a^{\prime}, b \leq b^{\prime}$ and $(a, b) \neq\left(a^{\prime}, b^{\prime}\right)$. The simplices of $(K \times L)^{\prime}$ are precisely the linearly ordered subsets, which we always write in increasing order.

Now the cross-product in homology is induced by the chain map $\left(Z_{2}\right.$ coefficients! $) C_{*}\left(K^{\prime}\right) \otimes C_{*}\left(L^{\prime}\right) \rightarrow C_{*}\left((K \times L)^{\prime}\right)$ given by

$$
\left\langle a_{0} \cdots a_{p}\right\rangle \otimes\left\langle b_{0} \cdots b_{q}\right\rangle \mapsto \sum\left\langle\left(a_{i_{0}}, b_{j_{0}}\right) \cdots\left(a_{i_{p+q}}, b_{j_{p+q}}\right)\right\rangle
$$

where the sum is over all pairs $i_{0} \leq \cdots \leq i_{p+q}, j_{0} \leq \cdots \leq j_{p+q}$ such that $\left\{i_{0}, \cdots, i_{p+q}\right\}=\{0, \cdots, p\},\left\{j_{0}, \cdots, j_{p+q}\right\}=\{0, \cdots, q\}$, and for each $r$, either $i_{r}<i_{r+1}$ or $j_{r}<j_{r+1}$.

Let $\sigma=\left\langle\left(a_{0}, b_{0}\right) \cdots\left(a_{p}, b_{p}\right)\right\rangle\left\langle(K \times L)^{\prime}\right.$. We say that $\sigma$ has a jump at $i$ if $a_{i-1}<a_{i}$ and $b_{i-1}<b_{i}$. Then clearly $\sum_{r} w_{r}(X) \times w_{p-r}(Y)$ is rep- 
resented by the sum of all $\sigma^{p}<(K \times L)^{\prime}$ such that $\sigma^{p}$ has no jumps. Now by the Proposition below, $w_{p}(X \times Y)$ is represented by $s_{p}\left((K \times L)^{\prime}\right)=$ sum of all $\sigma^{p}<(K \times L)^{\prime}$. Thus if

$$
c_{p}=s_{p}\left((K \times L)^{\prime}\right)-\sum_{r} s_{r}\left(K^{\prime}\right) \times s_{p-r}\left(L^{\prime}\right),
$$

then $c_{p}=$ sum of all $\sigma^{p}<(K \times L)^{\prime}$ such that $\sigma^{p}$ has at least one jump, and the product formula is equivalent to the fact that $c_{p}$ is a boundary.

We construct an explicit chain $d_{p+1}$ such that $\partial d_{p+1}=c_{p}$. First we say that $\left(a_{i}, b_{i}\right)$ is a critical vertex of $\tau=\left\langle\left(a_{0}, b_{0}\right) \cdots\left(a_{p+1}, b_{p+1}\right)\right\rangle$ if either

(i) $a_{i-1}<a_{i}=a_{i+1}$ and $b_{i-1}=b_{i}<b_{i+1}$, or

(ii) $a_{i-1}=a_{i}<a_{i+1}$ and $b_{i-1}<b_{i}=b_{i+1}$.

We then define an integer $(\bmod 2), \nu(\tau)$, by $\nu(\tau)=$ number of critical vertices of $\tau$ of type (i) before the first jump. (If $\tau$ has no jumps, $\nu(\tau)$ is the number of critical vertices of type (i).) Let

$$
d_{p+1}=\sum_{\tau^{p+1}<(K \times L)^{\prime}} \nu\left(\tau^{p+1}\right) \tau^{p+1} .
$$

Claim. $\partial d_{p+1}=c_{p}$.

Proof. $\partial d_{p+1}=\Sigma_{\sigma} \lambda_{\sigma} \sigma$ where

$$
\lambda_{\sigma}=\sum_{\tau^{p+1}>\sigma} \nu\left(\tau^{p+1}\right)
$$

For each $\sigma=\left\langle\left(a_{0}, b_{0}\right) \cdots\left(a_{p}, b_{p}\right)\right\rangle$, the $(p+1)$-simplices containing it are of the form

$$
\sigma_{i ; a, b}=\left\langle\left(a_{0}, b_{0}\right) \ldots\left(a_{i-1}, b_{i-1}\right)(a, b)\left(a_{i}, b_{i}\right) \ldots\left(a_{p}, b_{p}\right)\right\rangle
$$

(with the obvious definition of $\left.\sigma_{0 ; a, b}, \sigma_{p+1 ; a, b}\right)$. Then $\lambda_{\sigma}=\sum_{i=0}^{p+1} n_{i}(\sigma)$ where

$$
n_{i}(\sigma)=\sum_{\left(a_{i-1}, b_{i-1}\right)<(a, b)<\left(a_{i}, b_{i}\right)} \nu\left(\sigma_{i ; a, b}\right) .
$$

In the computations that follow, we constantly use the fact that if $x<y$ are vertices of $K^{\prime}$ or $L^{\prime}$, then each of the conditions $z<x, x<z<y, y<z$ is satisfied by an even number of vertices $z$. (Cf. Lemmas 1 and 2 below.)

Let $k \geq 1$ be the first jump of $\sigma$. (Possibly $k=p+1$, i.e., $\sigma$ has no jumps.) First we show that $\lambda_{\sigma}=n_{k}(\sigma)$, i.e., $n_{i}(\sigma)=0$ for $i \neq k$.

Consider the following cases: 
(1) $i>k$. Then $\nu\left(\sigma_{i ; a, b}\right)=\nu(\sigma)$ for all $(a, b)$, and the number of $\sigma_{i ; a, b}$ is even. Thus $n_{i}=0$.

(2) $i=0$. Divide the $\sigma_{0 ; a, b}$ onto three disjoint subsets according to the following conditions: $a<a_{0}, b<b_{0} ; a=a_{0}, b<b_{0} ; a<a_{0}, b=b_{0}$. In each subset, $\nu\left(\sigma_{0 ; a, b}\right)$ is constant, and the number of $\sigma_{0 ; a, b}$ in each subset is even. Hence $n_{0}=0$.

(3) $0<i<k$. In this case either $a_{i-1}=a_{i}$ and $b_{i-1}<b_{i}$ or $a_{i-1}<$ $a_{i}$ and $b_{i-1}=b_{i}$. If the first condition holds, then for all $\sigma_{i ; a, b}, a_{i-1}=$ $a=a_{i}$ and $b_{i-1}<b<b_{i}$. Thus $\nu\left(\sigma_{i ; a, b}\right)$ depends only on $i$ (and similarly if the second condition holds). Hence, as above, $n_{i}=0$.

Since $\lambda_{\sigma}=n_{k}$, the claim will be proved by showing that $n_{k}=1$ if $\sigma$ has jumps and 0 if $\sigma$ has no jumps, i.e., it has to be shown that $n_{k}=1$ if $k \leq p$ and $n_{k}=0$ if $k=p+1$.

Suppose $k=p+1$. Then $\nu\left(\sigma_{p+1 ; a, b}\right)$ is constant on each of the three subsets $a_{p+1}<a, b_{p+1}<b ; a_{p+1}=a, b_{p+1}<b ; a_{p+1}<a, b_{p+1}=b$; and there is an even number of vertices in each subset. Hence $n_{k}=0$ in this case.

Suppose $k \leq p$. Then $\nu\left(\sigma_{k ; a_{k}, b_{k-1}}\right)=\nu\left(\sigma_{k ; a_{k-1}, b_{k}}\right)+1$, and $\nu$ is again constant on each of the subsets $\left(a, b_{k-1}\right) ;\left(a_{k-1}, b\right) ;(a, b)$ with $a_{k-1}<a<a_{k}, b_{k-1}<b<b_{k}$. Hence in this case $n_{k}=1$.

The proof of the Claim is now complete.

2. Ordered triangulations. By an ordered triangulation of a polyhedron $X$, we mean a triangulation $K$ of $X$ together with a partial ordering on the vertices such that the simplices of $K$ are precisely the linearly ordered subsets (e.g., the first barycentric subdivision of a cell complex). We always write the simplices of $K$ in increasing order.

If $a$ is a vertex of $K$, we write simply $a^{-}$for $\langle a\rangle_{0}=\{b: b<a\}$, and $a^{+}$for $\langle a\rangle_{1}=\{b: a<b\}$. Let $|a|=1+\operatorname{dim} a^{-}$. If $K$ is a finite complex, $\#(K)$ means the number of vertices of $K$.

Note that if $K$ is an ordered triangulation, then we can define the Stiefel chains $s_{p}(K) \in C_{p}(K, \mathbf{Z})$ by the same formula (1).

If $\sigma=\left\langle a_{0} \cdots a_{p}\right\rangle<K$, then $L k(\sigma, K)=\sigma_{0} * \sigma_{1} * \cdots * \sigma_{p+1}$, where $\sigma_{i}$ is the full subcomplex of $K$ spanned by the vertices $a$ such that $a_{i-1}<a$ $<a_{i}$.

Lemma 1. Let $K$ be an ordered triangulation of an integral (resp. mod 2) Euler space, and let $\sigma<K$. Then each $\sigma_{i}$ is an integral (resp. mod 2) Euler space and $\chi\left(\sigma_{i}\right)=1+(-1)^{\operatorname{dim} \sigma_{i}}\left(\right.$ resp. $\chi\left(\sigma_{i}\right)$ is even $)$. 
Proof. It is easy to see that $L k(\sigma, K)$ is an Euler space, and hence that each $\sigma_{i}$ is an Euler space. Since $\sigma_{i}$ is the link of a maximal simplex in the join of the remaining $\sigma_{j}$ 's, it follows that $\sigma_{i}$ has the Euler characteristic of a sphere of the same dimension (cf. [2, \$2]).

Lemma 2. If $K$ is an ordered triangulation of a compact integral (resp. mod 2) Euler space, then $\chi(K)=\Sigma_{a \in K}(-1)|a|(\operatorname{resp} . \quad \chi(K) \equiv(\#(K)(\bmod 2))$.

Proof. Note that

$$
\begin{aligned}
\chi(K) & =\sum_{p}(-1)^{p}\left(\text { number of } p \text {-simplices }\left\langle a_{0} \cdots a_{p}\right\rangle\right) \\
& =\sum_{a}\left\{\sum_{p \geq 1}\left[(-1)^{p}\left(\text { number of }(p-1) \text {-simplices in } a^{-}\right)\right]+1\right\} \\
& =\sum_{a}\left(1-\chi^{\left.\left(a^{-}\right)\right) .}\right.
\end{aligned}
$$

But by Lemma $1,1-\chi\left(a^{-}\right)=(-1)^{|a|}\left(\right.$ resp. $\left.1-\chi\left(a^{-}\right) \equiv 1(\bmod 2)\right)$.

Lemma 3. If $K$ is an ordered triangulation of an integral Euler space, then

$$
\partial s_{p}(K)=\left(1+(-1)^{n-p}\right) s_{p-1}(K)
$$

Proof. By Lemmas 1 and 2, this is the same as the proof of Proposition 1 of [2], replacing $L k\left(a_{i}, a_{i+1}\right)$ by $\sigma_{i+1}$.

Proposition. Let $K$ be an ordered triangulation of $X$.

(i) If $X$ is a mod-2 Euler space, then $s_{p}(K)$ represents $w_{p}(X) \in$ $H_{p}\left(X, \mathbf{Z}_{2}\right)$.

(ii) Moreover, if $X$ is an integral Euler space and $n-p$ is odd, $p>0$, then $s_{p}(K)$ represents $w_{p}(X) \in H_{p}(X, \mathbf{Z})$.

Proof. By Lemma 3, (ii) follows from (i). To prove (i), define a simplicial map $\phi: K^{\prime} \rightarrow K$ as follows. If $\sigma<K$ and $\hat{\sigma}$ denotes its barycenter, let $\phi(\hat{\sigma})=m(\sigma)$ where $m(\sigma)$ is the maximum vertex of $\sigma$. $\phi$ extends to a simplicial map that induces the identity in homology.

Now an easy computation gives ( $Z_{2}$ coefficients!)

$$
\phi\left(s_{p}\left(K^{\prime}\right)\right)=\sum_{a_{0}<\cdots<a_{p}} \alpha_{a_{0} \cdots a_{p}}\left\langle a_{0} \cdots a_{p}\right\rangle
$$

where, if $\sigma=\left\langle a_{0} \cdots a_{p}\right\rangle$, 


$$
a_{a_{0} \cdots a_{p}}=\left(\#\left(\sigma_{0}\right)+1\right)\left(\#\left(\sigma_{1}\right)+1\right) \cdots\left(\#\left(\sigma_{p+1}\right)+1\right) .
$$

But by Lemma $2, \#\left(\sigma_{i}\right) \equiv \chi\left(\sigma_{i}\right)$, which is even by Lemma 1 . Thus $a_{a_{0} \ldots a_{p}}$ $\equiv 1$ and $\phi\left(s_{p}\left(K^{\prime}\right)\right)=s_{p}(K)$.

The first part of the Proposition was the only step missing in the proof of the Theorem. To prove the Corollary, let $\times: C_{*}\left(K^{\prime}, \mathbf{Z}\right) \otimes C_{*}\left(L^{\prime}, \mathbf{Z}\right) \rightarrow$ $C_{*}\left((K \times L)^{\prime}, \mathbf{Z}\right)$ be any chain map inducing the cross-product in homology; i.e., such that $\partial(x \times y)=(\partial x) \times y+(-1)^{p} x \times \partial y$ if $x \in C_{p}\left(K^{\prime}, \mathbf{Z}\right)$. For example, we could use the same formula (2) with appropriate signs.

An easy computation then shows that if $m+n-p$ is odd,

$$
\partial\left(\sum_{r=0}^{p+1} s_{r}\left(K^{\prime}\right) \times s_{p+1-r}\left(L^{\prime}\right)\right)=2 \sum_{r=0}^{p}(-1)^{m r_{s}}{ }_{r}\left(K^{\prime}\right) \times s_{p-r}\left(L^{\prime}\right) .
$$

Thus $\sum_{r=0}^{p}(-1)^{m r} s_{r}\left(K^{\prime}\right) \times s_{p-r}\left(L^{\prime}\right)$ is an integral cycle that represents the Bockstein of $w_{p+1}(X \times Y)$.

\section{REFERENCES}

1. E. Akin, Stiefel-Whitney homology classes and bordism, Trans. Amer. Math. Soc. (to appear).

2. S. Halperin and D. Toledo, Stiefel-Whitney homology classes, Ann. of Math. (2) 96 (1972), 511-525. MR 47 \# 1072.

3. D. Sullivan, Combinatorial invariants of analytic spaces, Proc. Liverpool Singularities Sympos. I (1969/70), Lecture Notes in Math., vol. 192, Springer-Verlag, Berlin, 1971, pp. 165-168. MR 43 \#4063.

4. H. Whitney, On the theory of sphere bundles, Proc. Nat. Acad. Sci. U.S.A. 26 (1940), 148-153. MR 1, 220.

DEPARTMENT OF MATHEMATICS, UNIVERSITY OF TORONTO, TORONTO, ONTARIO, CANADA (Current address of Stephen Halperin)

SCHOOL OF MATHEMATICS, INSTITUTE FOR ADVANCED STUDY, PRINCETON, NEW JERSEY 08540

Current address (Domingo Toledo): Department of Mathematics, Columbia University, New York, New York 10027 